\title{
Quantifying QoE of Mobile Video Consumption in a Real-Life Setting Drawing on Objective and Subjective Parameters
}

\author{
T. De Pessemier ${ }^{1}$, K. De Moor ${ }^{2}$, A. Juan ${ }^{1}$, W. Joseph ${ }^{1}$, Member, IEEE, \\ L. De Marez ${ }^{2}$, L. Martens ${ }^{1}$, Member, IEEE
}

\begin{abstract}
The ability to quantify Quality of Experience (QoE, i.e. the users' subjective perception of the 'overall acceptability of an application or service'), will play a major role in the success of future multimedia services such as mobile video watching. Such QoE-evaluations are however still challenging, especially in reallife (or so-called 'Living Lab'), user settings. According to our knowledge, this paper is the first to present a methodology for the multi-dimensional quantification of $\mathrm{QoE}$ during mobile video watching in real-life settings. A measurement study drawing on the evaluation of objective and subjective QoE aspects by a user panel resulted in a model for quantifying QoE during mobile video watching based on user behavior and technical parameters such as network and video quality.
\end{abstract}

Index Terms - mobile video, Quality of Experience, user experiment, objective evaluation techniques, subjective evaluation techniques, Living Lab.

\section{INTRODUCTION}

$\mathrm{I}^{\mathrm{N}}$ $\mathrm{N}$ the mobile communications domain, characterized by a heterogeneity and increasing offer of services and applications, users' Quality of Experience (QoE) has become a crucial determinant of the success or failure of new applications and services. QoE has been defined by ITU as 'the overall acceptability of an application or service, as perceived subjectively by the end-user' [1], and this QoE can be influenced by various aspects, such as subjective human factors (e.g. expectations), contextual factors, and technical performance parameters [2]. It is increasingly argued that the ability to understand, quantify and optimize QoE in various user contexts will play a major role in the design of future multimedia services, not the least in the dynamic mobile media domain [3].

Manuscript received May 6, 2011. We would like to thank the Research Foundation-Flanders (FWO), for the research position of Toon De Pessemier (predoctoral fellow) and Wout Joseph (post-doctoral fellow). This work was supported by the IBBT / UGent (Interdisciplinary institute for BroadBand Technology / Ghent University) through the GR@SP-project.

${ }^{1}$ T. De Pessemier, A. Juan, W. Joseph and L. Martens are with the Department of Information Technology, Ghent University/IBBT, Gaston Crommenlaan 8, B-9050 Ghent, Belgium. (email: toon.depessemier@intec.ugent.be phone:+32 933 14908)

${ }^{2}$ K. De Moor L. De Marez are with the Department of Communication Sciences, Ghent University/IBBT, Korte Meer 7-9-11. B-9000 Ghent, Belgium
From the viewpoint of the user, the multi-dimensional character of human experiences and the influence of contextual variables upon the interaction with ICT products is emphasized. From a network and performance perspective on the other hand, there is a need for quantified insights and clear threshold values. Both perspectives can be seen as complementary. Yet, the integration of knowledge and expertise from these different traditions and perspectives is still ongoing.

The objective of this paper is to quantify the QoE of mobile video consumption in a real-life setting based user behavior and technical parameters such as network and video quality. The remainder of the paper is organized as follows: Section 2 reviews existing work on QoE and places this research in the wider context of QoE. Section 3 is dedicated to the mobile video experiment and provides an overview of the logged subjective and objective data. A model for quantifying the QoE during mobile video watching is provided in Section 4. The conclusion of this user study is provided in Section 5.

\section{RELATED WORK}

Previous work on correlating network QoS and QoE mainly focus on the audiovisual quality. As a result, these studies do not cover all aspects that influence the QoE (like loading or rebuffering times). The influence of network loss and burst size on the audiovisual quality and acceptability of streaming video is studied by using subjective measurements in [4]. In this research, the subjective test showed that increasing loss levels result in poorer user opinions of both quality and acceptability. The impact of data loss on the quality of MPEG2 video was investigated in [5]. This study also analyzed how the user-perceived quality is related to the average encoding bitrate of the video. In [6], the impact of both jitter and packet loss on perceptual quality of packet video are measured and compared. This research found that jitter degrades perceptual quality nearly as much as does packet loss, and that perceptual quality degrades sharply even with low levels of jitter or packet loss as compared to perceptual quality for perfect video.

These studies are usually based on standardized assessment methods for audiovisual quality evaluation as e.g. described in [7] and [8]. These assessment methods are used for quantifying and simulating users' quality perceptions in order 
to optimize the relevant technical parameters. This type of research makes it possible to investigate the relative influence of particular isolated parameters on users' quality perceptions. However, the external validity of such controlled environment tests is highly questionable if the focus is on the assessment of users' experiences and quality perceptions concerning mobile applications or services. Here, the lack of realistic and natural contexts-of-use can be considered as problematic. In this paper, we therefore focus on the possible relevance and complementarities of more realistic, living lab test environments.

In the past, various studies on mobile video quality assessment were set up. A lot of them focused on mobile television. An interesting result is that the offered picture ratio mainly has an impact on the legibility of textual material and the ability to detect small details [9]. Moreover the significance of audio in low bitrates was emphasized. Examples in which the extension towards more natural and realistic research environments was made by organizing field trials or conducting living lab studies can also be found in the literature [10], [11].

As it is still largely unknown how objective network parameters, i.e. Quality of Service (QoS) [12], technical video properties and user contexts can be used to measure, estimate, and optimize QoE related to mobile video watching [13], the main objective of this paper is to quantify QoE related to mobile video watching using mobile telecommunications technologies. Objective measurements of technical parameters were performed by means of a mobile agent implemented on the test devices. Additionally, subjective evaluations were gathered by means of a questionnaire on the device, displayed immediately after a viewing session and self-reporting collected using traditional paper diaries. This paper proposes a methodology for linking users' subjective quality and experience assessment, behavior information, and technical parameters of the network and video (which are objectively measured during video watching). The exploratory Living Lab experiment described in this paper resulted in a QoE model that can be used for predicting and optimizing the users' experience during mobile video watching.

\section{MOBILE VIDEO EXPERIMENT}

\section{A. $\quad$ Test Setup}

Given the possible influence of contextual factors on users' QoE, there is a need for studying this QoE in real-life and realistic settings. In literature, the 'Living Lab' concept is pushed forward in this respect. A Living Lab is defined by [14] as "environment for innovation and development where users are exposed to new ICT solutions in (semi) realistic contexts, as part of medium- or long-term studies targeting evaluation of new ICT solutions and discovery of innovation opportunities". Since Living Labs draw on real people's experiences, QoE research in such settings will likely yield more accurate results and have a higher validity than research in controlled environments [15], [16].

For this exploratory Living Lab experiment, 29 test users,

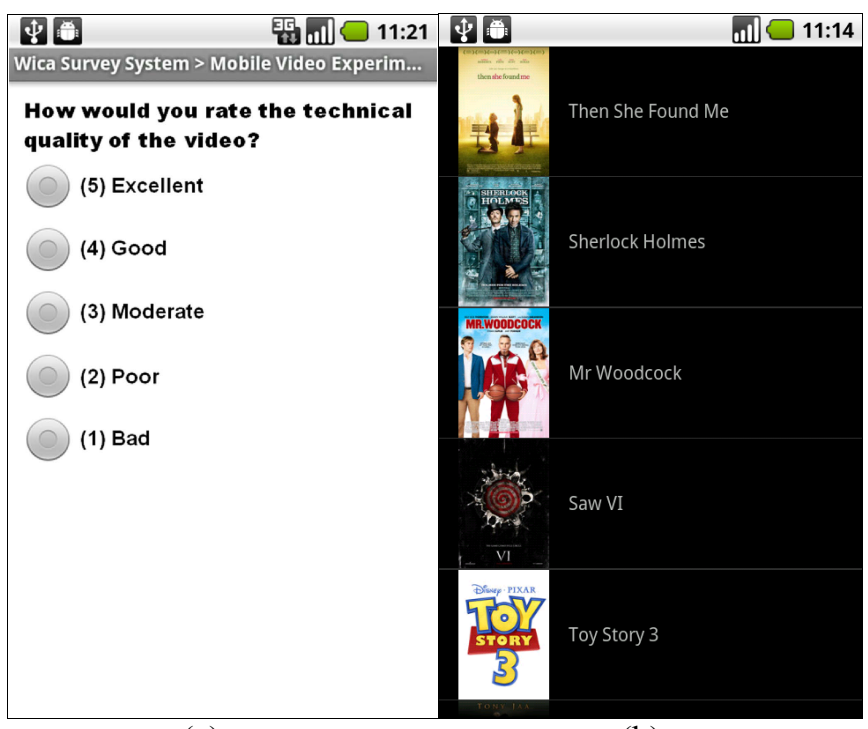

(a)

(b)

Fig. 1. Screenshots of the video application on the mobile device. The left part (a) illustrates the questionnaire, the right part (b) shows the video selection mechanism.

who are experienced smart phone users and indicated in a preceding recruitment survey that they are interested in video content, were asked to watch 28 videos on a mobile device (Nexus One phone running Android 2.1) in their natural environment. After watching a clip, they were asked to answer a few questions (on a 5-point star rating scale) to evaluate the audiovisual aspects and the QoE on the device (Figure 1a). Before the test, every user received instructions in a preceding instruction meeting. After the general information on how to switch on/off, use, and charge the device, it was explained how to access the test application and how to select and watch the videos. Next, it was also shown how to fill in the questionnaire using the touch screen. At the end of the briefing session, every test user was given a device, a diary (to provide additional feedback) and an instruction leaflet with practical information, screenshots, and relevant instructions related to the grading scales and univocal interpretation of the questions. In total, 6 instruction meetings were organized (in groups of five test users). The data gathering phase took just over three months since five Nexus One devices rotated among the test users.

Every test user had one full week time to watch all the videos. Since we aimed to explore the actual viewing behavior in a natural context-of-use, the respondents could decide themselves when and where they watched the videos. One test user who pulled out before the actual test period due to time constraints, was not replaced. So 29 people (22 male and 7 female) between 20 and 61 years old (mean $=33.10$, standard deviation $=9.97)$ participated in the study. This yielded 812 samples, providing enough data to develop a QoE model for mobile video watching. Every test user received a gift voucher of 10 Euro as incentive. The 28 videos, a fixed, predefined list of movie trailers covering different genres (as visualized in Figure $1 b$ ), had a duration between 2 and 3 minutes. 


\section{B. Video types: quality and transport protocol}

To investigate the influence of the video quality and the transport protocol on the QoE, the video list consisted of 4 categories: 7 low-quality videos using RTP (Real-time Transport Protocol), 7 high-quality videos using RTP, 7 lowquality videos using progressive download (based on HTTP) and 7 high-quality videos using progressive download.

Both RTP and progressive download are often used but have different characteristics in terms of possible influence on the user's experience. RTP defines a standardized packet format for delivering audio and video over IP networks and is used extensively in communication and entertainment systems that involve streaming media, like teleconference applications. These streaming media require timely delivery of information and allow no retransmissions in case of packet loss, which may lead to noticeable distortions for the user. Progressive download is a technique used to transfer digital media files, in which users may begin playback of the media before the download is complete. For transferring media, progressive download is typically using HTTP (Hypertext Transfer Protocol) which is based on a reliable transport layer protocol for host-to-host data transfer, in most cases TCP (Transmission Control Protocol). Since TCP guarantees a reliable, ordered delivery of a stream of data by using packet retransmissions and buffers, no video distortion will occur. However, packet delay or retransmissions may cause rebuffering interruptions of the media player [17].

TABLE I. TECHNICAL PARAMETERS OF THE MOBILE VIDEO

\begin{tabular}{|c|c|c|c|}
\hline \multicolumn{4}{|c|}{ Low Quality Video } \\
\hline Audio & & Video & \\
\hline Bandwidth & $32 \mathrm{Kbit} / \mathrm{s}$ & Bandwidth & $128 \mathrm{Kbit} / \mathrm{s}$ \\
\hline Channels & 2 & Resolution & $142 * 80$ \\
\hline Codec & AAC LC & Codec & H.264/AVC \\
\hline $\begin{array}{l}\text { Sampling } \\
\text { frequency }\end{array}$ & $44100 \mathrm{~Hz}$ & Framerate & $24 \mathrm{fps}$ \\
\hline \multicolumn{4}{|c|}{ High Quality Video } \\
\hline Audio & & Video & \\
\hline Bandwidth & $128 \mathrm{Kbit} / \mathrm{s}$ & Bandwidth & $384 \mathrm{Kbit} / \mathrm{s}$ \\
\hline Channels & 2 & Resolution & $512 * 288$ \\
\hline Codec & AAC LC & Codec & H.264/AVC \\
\hline $\begin{array}{l}\text { Sampling } \\
\text { frequency }\end{array}$ & $44100 \mathrm{~Hz}$ & Framerate & $24 \mathrm{fps}$ \\
\hline
\end{tabular}

These transfer protocols were combined with two video qualities to investigate their impact upon the user's quality evaluation and to explore whether users prefer a more fluent video playback (even if this implies lower video quality) or whether they prefer a higher video quality (implying rebuffering interruptions and possible image distortion). Technical details concerning the two video quality versions that were used in the test are provided in Table I. Test users, not informed about the variable quality or transport protocol, received a randomly-mixed list of videos (as illustrated in Figure 1b). However, a statistical T-test proved the different qualities $(\mathrm{p}=0.0)$ and transport protocols $\left(\mathrm{p}=9.0 \cdot 10^{-4}\right)$ can be distinguished by the user, resulting in varying experiences.

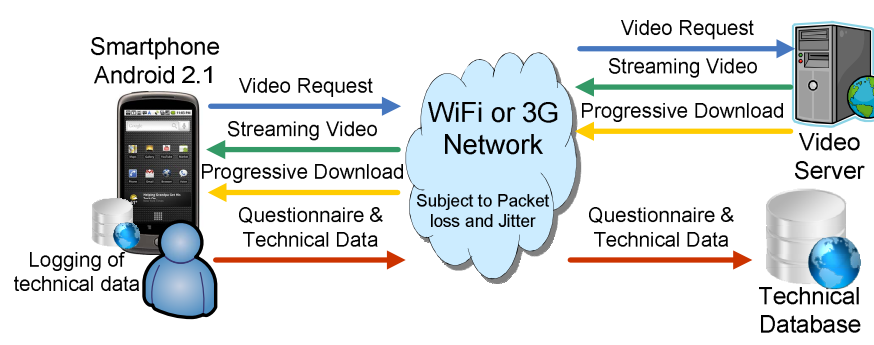

Fig. 2. Architecture of the video delivery system.

\section{Data Logging}

The mobile video measurement is performed according to the context-aware experience sampling method in a commercial UMTS / HSDPA (Universal Mobile Telecommunications System / High Speed Downlink Packet Access) network in Belgium. Figure 2 shows the architecture of the video delivery system consisting of the client (i.e. the smartphone running the video player), video server and technical database.

While playing a video, the following technical, objective parameters regarding the video and network connection are logged: video resolution, transport protocol (RTS or progressive download), packet-loss rate for the audio and video track, the mean and maximum jitter (i.e., the variability over time of the packet latency across the network) for audio and video, network type (e.g., UMTS, HSDPA, GPRS), number of handovers (i.e., all kinds of radio cell reselections) and inter-system handovers (i.e., different data connectiontype cell reselections e.g., between UMTS and HSDPA), and RSSI (received signal strength indicator).

In addition, objective parameters about the video session and watching behavior are registered: movement of the device (i.e., the GPS signal to track the mobility during the video watching), early interruption of the video (e.g., due to network disconnection or a disinterest of the user), metadata about the video (id, title, length) and the start and end of the session (timestamp).

As mentioned above, users were also asked to fill in a questionnaire on the device, immediately after each video watching session. This subjective evaluation assesses the user's appreciation of the content and his/her opinion concerning the loading speed, fluentness, general experience, and noticeable distortion in the watched video. A question about the physical context of the test user ("Are you at home, on the move, at work, or somewhere else?"), together with the objectively logged movements of the device, provide information about the usage context. Additionally, extra feedback was collected in the user diaries.

The collected data were cleaned and integrated into one data file containing the logged technical data, the subjective ratings collected through the questionnaires on the device and the paper diary entries for every question. In this paper, we zoom in on one particular aspect, i.e., the subjective evaluation of the user's experience during the video watching session. After 


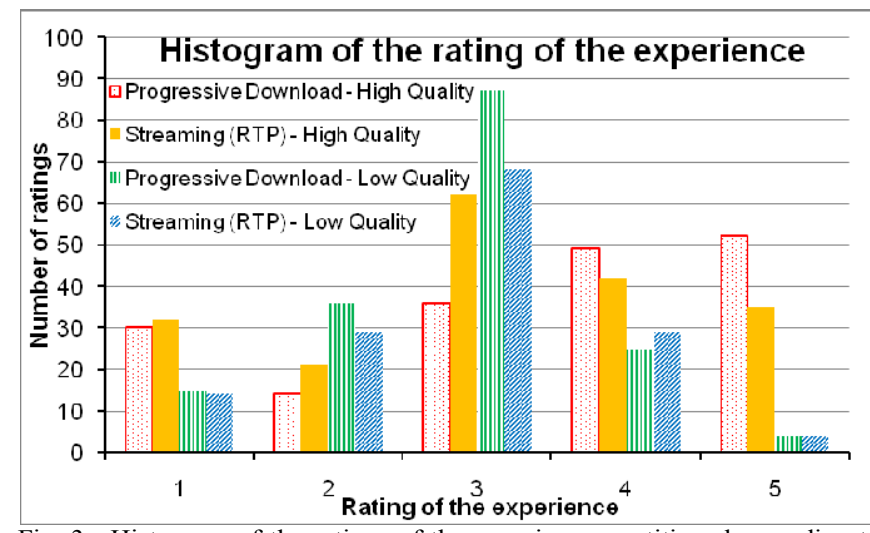

Fig. 3. Histogram of the ratings of the experience, partitioned according to the transport protocol and the quality of the video source.

excluding a small number of data samples (for which the question regarding the experience was not answered), the data set consisted of 684 subjective evaluations of user's experience together with the technical parameters of the video session.

\section{Modeling THE QoE}

\section{A. Distribution of the experience ratings}

Figure 3 visualizes the histograms of the ratings provided by the test users regarding their general experience during the video watching session. This histogram shows that the majority of the video sessions rated with an experience value of 4 or 5 stars, are based on a high quality video source. In contrast, the majority of the videos sessions characterized by a poor or moderate QoE (2 or 3 stars) are based on a low quality video source.

Furthermore, the histogram indicates that more video sessions which received an excellent experience evaluation (5 stars) are using progressive download, than using streaming (RTP). The ability of progressive download to avoiding packet loss by packet retransmissions, might lead to a better QoE.

However, the quality of the video source and the transport protocol alone are not sufficient to explain all experience values. E.g. video sessions with a very bad experience (1 star) are mostly using high quality video sources. This bad experience might be due to a limited bandwidth causing annoying video distortions, extra rebuffering times or video interruptions. Since high quality videos require more bandwidth; distortions, rebufferings, and interruptions have a higher probability of occurrence during the transmitting of these high quality videos. In case of a limited bandwidth (e.g. while using a GPRS connection), low quality videos might be more robust for these distortions and rebufferings, leading to a better QoE than the high quality videos.

As a result, a thorough statistical analysis is required to model the QoE during a mobile video session based on the technical parameters of the video and the transport protocol, but also the network parameters, and the watching behavior.

\section{$B$. Training and evaluating the decision tree}

The QoE of the end-user during video watching on the mobile device can be obtained by assessing the subjective experience of the user through a questionnaire, like in this research experiment. However, since such a questionnaire might be too intrusive and not desirable in commercial applications, the measurement or estimation of QoE must be based on the available technical properties, obtained without user interference. In this experiment, we modeled the user's QoEe, obtained via the questionnaire, based on the technical parameters of the video, the transport protocol, the network parameters, and the watching behavior of the user. This model of the user's experience is obtained via a decision tree, a classification technique that uses a tree-like graph or model of decisions and their possible consequences [18]. This decision support tool is generally preferred over other nonparametric techniques because of the readability of their learned hypotheses and the efficiency of training and evaluation.

The minimum set of technical parameters required to model the QoE was determined through a statistical analysis. The resulting parameters regarding the video session that are used as input for the decision tree are: the transport protocol, the quality of the video source, the types of data network that were used to transmit the video, the number of handovers during transmission, and the percentage of the video that was actually watched by the user. Because of the mutual correlation of the technical parameters, additional parameters, like packet loss, jitter, mobility of the user, and signal strength of the network, have no additional information value and do not further improve the classification model; i.e. the inclusion of these parameters in the decision tree did not lead to a further decrease of the misclassification rate.

TABLE II. MisclassSifiCATIONRATE OF THE DECISION TREE

\begin{tabular}{cc}
\hline & Ratio of test samples \\
\hline Correct classification & $46.2 \%$ \\
\hline 1 star misclassifcation & $35.3 \%$ \\
\hline 2 stars misclassifcation & $16.2 \%$ \\
\hline 3 stars misclassifcation & $1.6 \%$ \\
\hline 4 stars misclassifcation & $0.7 \%$ \\
\hline
\end{tabular}

To train the decision tree, $90 \%$ of the data samples were used as training set (i.e. 616 randomly selected samples); the remaining 10\% (68 samples) constitutes the test set and was utilized for the validation of the decision tree. After training the decision tree, the samples in the test set were classified according to the obtained decision model and the misclassification rate was calculated. This procedure was repeated 20 times to eliminate influences of the random data partitioning in training and test set. The averages of the misclassification rates, obtained during these 20 iterations, are indicated in Table II. For almost half of the test samples (46.2\%), the decision tree is able to correctly predict the QoE rating provided by the user based on the watching behavior, transport protocol and the technical parameters of the video and the network. Moreover the QoE of $81.6 \%$ of the video sessions is classified correct or within an acceptable error margin of 1 star. The ratio of severe misclassifications (i.e. 3 


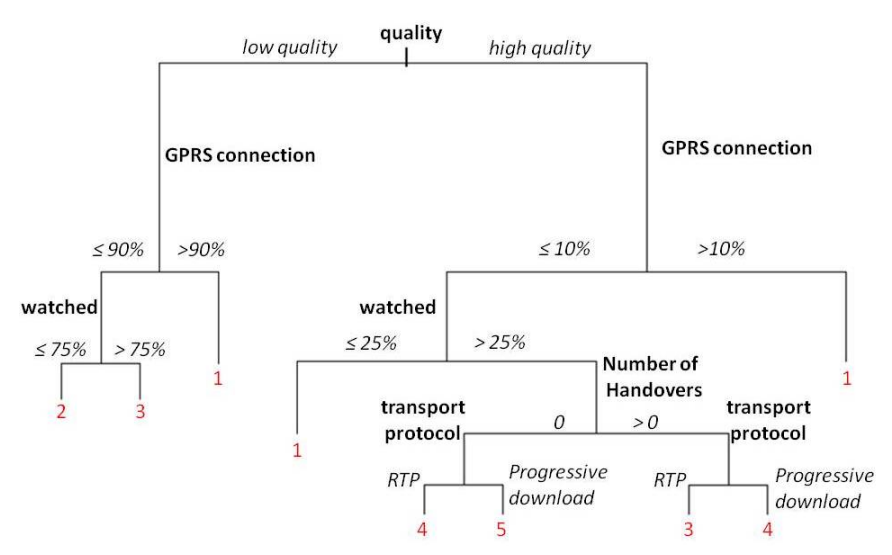

Fig. 4. Decision tree modeling the QoE during video watching on a mobile device based on the watching behavior and the technical parameters of the video and network.

or 4 stars deviance between the predicted experience and the actual experience) is limited to $2.3 \%$ of the samples, which proofs the usefulness of the decision tree.

\section{Predicting the experience based on the decision tree}

Figure 3 shows the visualization of the decision tree, which can be used to predict the user's experience based on the technical parameters of the video session. To avoid over fitting and limit the complexity of the model, the decision tree was pruned until a deviance ${ }^{1}$ of 0.008 was obtained. The starting point of this decision tree is the root, situated at the top of the figure.

At the first fork, a decision is made based on the quality of the video source: if the video source has a low quality, the left branch is chosen; the right branch is followed, in case of a high quality video source. These low quality videos typically induce a poor to moderate QoE: the experience ratings for low quality videos are ranging from 1 to 3 stars in the decision tree. To predict the QoE while watching low quality video sources, the type of data network used for transmitting the video, is important. If the video is mainly transmitted over a GPRS connection (more than $90 \%$ of the video is transmitted over a GPRS connection, so less than $10 \%$ is transmitted over a faster data connection), the decision tree predicts a $\mathrm{QoE}$ value of 1 star. The reason for this bad experience may be the combination of a low quality video source and a slow data connection type (GPRS). This slow data connection may introduce interruptions during video playback (i.e. long rebuffering times if progressive download is used or audiovisual distortions if the video is streamed). If the low quality video is (partly) transmitted over a faster data network, an additional criterion is investigated to estimate the user's experience.

The next branch is based on the part of the video that was actually watched by the user. On the one hand, if the user watched the video (almost) completely (more than 75\%), this may suggest that no network problems occurred during the video playback and the user experienced the video session as

\footnotetext{
${ }^{1}$ Prune on deviance is a measure that defines a stopping rule in the pruning process based on maximum-likelihood principles.
}

acceptable. In this situation, the user will rate her experience with 3 stars, according to the decision tree. On the other hand, a video session that is stopped early by the user (less than $75 \%$ of the video is watched) can indicate a bad experience due to an unacceptable audiovisual quality or a disinterest of the user for the content of the video. Alternatively, the video interruption might be due to a network problem (e.g. a network disconnection), which also induces a bad experience for the user. Therefore the decision tree provides a prediction of 2 stars for the QoE in this condition.

In case of a high quality video source, the right half of the decision tree is used to classify the QoE. A GPRS connection is basically too slow to transmit the high quality video, thereby introducing distortions or interruptions during video playback. So, if a GPRS network is used for (more than $10 \%$ of) the transmission of the high quality video, the QoE is not be acceptable, which is indicated by a prediction of 1 star for the experience value. The other branch represents the situation of transmitting a high quality video source over a fast data network (no or limited GPRS is used). In this situation, the QoE is predicted based on the fraction of the video that is actually watched by the user. Videos might be interrupted by the user because of a bad experience (due to a low audiovisual quality or uninteresting content). In addition, video interruptions due to network problems can be the cause of a bad QoE. As a result, these early video interruptions lead to a prediction value of 1 star for the QoE.

High quality videos that are transmitted over a fast network without an early video interruption (more than $75 \%$ of the video is watched) indicate a good QoE. In this situation, the experience value is predicted based on the used transport protocol and the number of handovers. High quality video sources streamed over the cellular data network may suffer from packet loss and jitter, inducing video distortions. Progressive download can resolve these packet losses and avoid distortions by using retransmission but may thereby introduce extra rebuffering times. The subjective evaluations of the user tests learned that users prefer videos without distortions, even if this implies some extra rebuffering moments. In other words, users have a better experience with videos transmitted using progressive download, than with streamed videos (using RTP). Therefore, the experience of video sessions using progressive download is predicted to be (1 star) better than the experience of video sessions based on streaming.

During a handover, an ongoing data session is transferred from one channel or cell to another one. Since this process may introduce an interruption of the data transmission, it can have an influence on the QoE. As a result, the decision tree predicts a lower experience value for video sessions with handovers than for sessions without handovers.

This decision tree can be used to predict and improve the user's QoE of a mobile video session. E.g., if a fast cellular data network is available for the transmission of half of the video, (i.e. $50 \%$ of the video is transmitted over a GPRS network and $50 \%$ is transmitted over a faster network like UMTS or HSDPA), a low quality video source might be 
preferred above a high quality video source. In this situation, the decision tree predicts an experience value of 1 star for the high quality video source, while the low quality video source receives 2 or 3 stars depending on the fraction of the video that is actually watched. In contrast, if a fast cellular data network is available for the complete transmission of the video (and video playback is not interrupted), a higher quality video source might result in a better QoE, as indicated in the decision tree.

\section{CONCLUSIONS}

In this paper, we discussed the results from an exploratory study on the user's QoE during mobile video watching. Test users were asked to watch 28 video trailers in random combinations of two video qualities (high and low) and two transport protocols for video (real-time transport protocol and progressive download using HTTP). Although users could watch the offered content where and when they wanted, we label this study as semi-living lab since the test users were 1) given an additional device to perform the test, 2) asked to watch a limited and pre-defined content list, 3) given one week time to finish the test. Subjective experience evaluations, gathered by means of short questionnaires on the device and traditional paper diaries, were combined with the logging of technical parameters regarding the video quality, transport protocol, network characteristics and watching behavior of the user.

Based on these data, a decision tree is created to quantify the QoE during mobile video watching. This model provides application developers and service providers a tool that clarifies which and how technical parameters influence the QoE and how the parameters have to be adapted to optimize the QoE. E.g. if no fast cellular data network is available (only a GPRS network is available), the model predicts a bad QoE for high quality video sources due to distortions or a large amount of rebufferings during video playback. In this case, low quality video sources might result in a better QoE. If a fast data network is available, a high quality video transmitted using progressive download provides an optimal QoE.

\section{REFERENCES}

[1] ITU-T Recommendation, Telecommunication standardization sector of ITU. "Series p: telephone transmission quality, telephone installations, local line networks" P.10/G.100 (2006)/Amd. 1, Jan. 2007

[2] J. Korhonen, U. Reiter, and E. Myakotnykh, "On the relative importance of audio and video in the presence of packet losses," Second International Workshop on Quality of Multimedia Experience (QoMEX), 2010, pp. 64-69, June 21-23, 2010.

[3] M. Fiedler, K. Kilkki, and P. Reichl, "09192 Executive summary -- from quality of service to quality of experience", Dagstuhl Seminar, Schloss Dagstuhl - Leibniz-Zentrum, Germany, May 5-8, 2009.

[4] D. Hands and M. Wilkins. "A Study of the impact of network loss and burst size on video streaming quality and acceptability", Lecture Notes in Computer Science, pp. 45-57, 1999.

[5] O. Verscheure, P. Frossard, and M. Hamdi. "MPEG-2 video services over packet networks: Joint effect of encoding rate and data loss on useroriented QoS", In Proc. NOSSDAV, 1998.
[6] M. Claypool and J. Tanner. "The effects of jitter on the peceptual quality of video". In Proc. 7th ACM international conf. on Multimedia. ACM, New York, NY, USA, 1999.

[7] ITU-T P.911, "Subjective audiovisual quality assessment methods for multimedia applications". Telecommunications UnionTelecommunication sector, 1998.

[8] VQEG, "Final report from the video quality experts group on the validation of objective models of video quality assessment", 2000 .

[9] S. Jumisko-Pyykkö, "I would like to see the subtitles and the face or at least hear the voice: Effects of Picture ratio and Audio-video Bitrate Ratio on Perception of Quality in Mobile Television", Multimedia Tools and Applications, vol. 36, (1-2), pp. 167-184, 2008.

[10] D. Schuurman, L. De Marez, et al. "Content and context for mobile television. Integrating trial, expert and user findings", Telematics and Informatics, vol 26 (3), pp. 293-305, 2010.

[11] S. Jumisko-Pyykkö and M.M. Hannuksela, "Does Context Matter in Quality Evaluation of Mobile Television?" In Proc. 10th International Conf. on Human Computer Interaction with Mobile Devices and Services, pp. 63-72, 2008

[12] ITU-T, Telecommunication standardization sector of ITU. "Series g: transmission systems and media, digital systems and networks quality of service and performance. end-user multimedia QoS categories", Nov. 2001.

[13] F. Agboma and A. Liotta, "QoE-aware QoS management", In Proc. 6th International Conf. on Advances in Mobile Computing and Multimedia (MoMM '08), pp. 111-116, ACM, New York, NY, USA, 2008.

[14] A. Følstad, "Living labs for innovation and development of information and communication technology: a literature review," The Electronic Journal for Virtual Organizations and Networks 'Special Issue on Living Labs' 10, pp. 99-131, 2008.

[15] J. Schumacher, K. Feurstein, O. Pitknen, and V. P. Niitamo, "European living labs: A new approach for human centric regional innovation," Wissenschaftlicher Verlag, Berlin, 2008.

[16] K. De Moor, I. Ketyko, W. Joseph, T. Deryckere, L. De Marez, L. Martens, and G. Verleye, "Proposed framework for evaluating quality of experience in a nobile, testbed-oriented living lab setting," Mobile Networks and Applications: Special Issue on Advances In Wireless Test beds and Research Infrastructures, vol. 15 (3), pp. 378-391, Springer Netherlands, June 2010

[17] R. Mok, E. Chan, and R. Chang, "Measuring the Quality of Experience of HTTP Video Streaming", In Proc. of the IFIP IM IEEE conference (Pre-conf Session), 2011.

[18] L. Breiman, J. Friedman, R. Olshen and C. Stone, "Classification and Regression Trees”, Wadsworth International Group, Belmont, CA, 1984. 